\title{
Elementary proof of the B. and M. Shapiro conjecture for rational functions
}

\author{
Alex Eremenko and Andrei Gabrielov*
}

October 10, 2005

\begin{abstract}
We give a new elementary proof of the following theorem: if all critical points of a rational function $g$ belong to the real line then there exists a fractional linear transformation $\phi$ such that $\phi \circ g$ is a real rational function. Then we interpret the result in terms of Fuchsian differential equations whose general solution is a polynomial and in terms of electrostatics.
\end{abstract}

One of the many equivalent formulations of the Shapiro conjecture is the following. Let $\mathbf{f}=\left(f_{1}, \ldots, f_{p}\right)$ be a vector of polynomials in one complex variable, and assume that the Wronski determinant $W(\mathbf{f})=W\left(f_{1}, \ldots, f_{p}\right)$ has only real roots. Then there exists a matrix $A \in \mathrm{GL}(p, \mathbf{C})$ such that $\mathbf{f} A$ is a vector of real polynomials.

This conjecture plays an important role in real enumerative geometry [15. 16], theory of real algebraic curves [8] and has applications to control theory [10, 4]. There is a substantial numerical evidence [16] in favor of the conjecture.

In [1] we proved the Shapiro conjecture in the first non-trivial case $p=2$. The proof was quite complicated, and its main drawback from the point of view of generalizations to higher dimensions was the use of the Uniformization theorem.

In this paper we give a new proof, not using the Uniformization theorem. The new proof is also much simpler than the arguments in [1].

*Both authors were supported by NSF. 
Consider a non-constant rational function $g=f_{1} / f_{2}$, and assume that the polynomials $f_{1}$ and $f_{2}$ are co-prime. Then the degree of $g$ is given by $d=$ $\max \left\{\operatorname{deg} f_{1}, \operatorname{deg} f_{2}\right\}$, and the roots of the Wronski determinant $W(\mathbf{f})=f_{1} f_{2}^{\prime}-$ $f_{1}^{\prime} f_{2}$ coincide with the critical points of $g$. Let us call two rational functions $g_{1}$ and $g_{2}$ equivalent if $g_{1}=\phi \circ g_{2}$ for some fractional-linear transformation $\phi$. Evidently, equivalent rational functions have the same critical points. So our result is

Theorem 1. If all critical points of a rational function are real then it is equivalent to a real rational function.

It is enough to prove this theorem for rational functions with simple critical points. The general case then follows by a limiting process.

It is known [6] that for given $2 d-2$ points in the complex plane in general position, there exist

$$
u_{d}=\frac{1}{d}\left(\begin{array}{c}
2 d-2 \\
d-1
\end{array}\right)
$$

the $d$-th Catalan number of classes of rational functions of degree $d$ with these critical points. It turns out that the general position assumption in this result can be removed if the given points are real. Moreover, the following result turns out to be equivalent to Theorem 1:

Theorem 2. For any given $2 d-2$ distinct points on the real line, there exist exactly $u_{d}$ distinct classes of rational functions of degree $d$ with these critical points.

It follows from Theorem 1 that each of these $u_{d}$ classes contains a real function. The assumption that the critical points are real is essential in Theorem 2: for $2 d-2$ complex points, the number of rational functions of degree $d$ with these critical points can be less than $u_{d}$.

Equivalence of theorems 1 and 2 was known for some time, see, for example, [16].

To state a generalization of Theorem 2 to the case of multiple critical points, we recall the definition of Kostka numbers. Let $\mathbf{a}=\left(a_{1}, \ldots, a_{q}\right)$ be a vector of integers satisfying

$$
1 \leq a_{j} \leq d-1, \quad \sum_{j=1}^{q} a_{j}=2 d-2 .
$$

Consider the Young diagrams of shape $2 \times(d-1)$. They consist of two rows 
of length $d-1$. A semi-standard Young tableau SSYT of shape $2 \times(d-1)$ is a filling of such a diagram by positive integers, such that an integer $k$ appears $a_{k}$ times, the entries are strictly increasing in columns and non-decreasing in rows. The corresponding Kostka number $K_{\mathbf{a}}$ is the number of such SSYT. The number $K_{\mathbf{a}}$ does not change if the coordinates of a are permuted [17, Thm. 7.10.2].

Theorem 3. For given a satisfying (2), and given real points $x_{1}<x_{2}<$ $\ldots<x_{q}$, there are exactly $K_{\mathbf{a}}$ classes of rational functions of degree $d$ with critical points at $x_{j}$ of multiplicity $a_{j}$.

We obtain Theorem 2 as a special case when $q=2 d-2$ and all $a_{j}=1$. Theorem 3 is true for generic complex $x_{j}$; this was derived by Scherbak [1] from the results in [19]. Theorem 3 was first proved in [5], where a result from [1] was used. We include a proof here to show that it can be achieved with the same elementary tools as theorems 1 and 2, and no heavy machinery from [1] is needed.

A self-contained proof of Theorems 1 and 2 is given in Section 1. In Section 2, we discuss two equivalent reformulations of these theorems, which in our opinion are of independent interest, and also might be helpful for proving the Shapiro Conjecture in higher dimensions. Many other reformulations of the Shapiro conjecture are contained in [13, 15, 16].

We thank Boris Shapiro, Frank Sottile and Alexander Varchenko for their useful comments on this paper.

\section{Proof of the main theorems}

\section{$1.1 \quad$ Wronski map}

We recall the necessary facts and definitions. Let $\mathbf{f}=\left(f_{1}, f_{2}\right)$ be a pair of linearly independent polynomials of degree at most $d$. They span a 2dimensional subspace in the space of all polynomials of degree at most $d$, and thus define a point in the Grassmannian $G=G(2, d+1)$. Two pairs of polynomials are equivalent if they span the same subspace.

The Wronski determinants of equivalent pairs are proportional non-zero polynomials of degree at most $2 d-2$. Classes of proportionality of such

polynomials form a space Poly ${ }^{2 d-2}$ which can be identified with the projective 
space $\mathbf{P}^{2 d-2}$. So taking Wronski determinant defines a map

$$
W: G(2, d+1) \rightarrow \text { Poly }^{2 d-2}
$$

which is called the Wronski map. The real Grassmannian $G_{\mathbf{R}}$ or the real projective space Poly $_{\mathbf{R}}$ consist of those points whose coordinates (coefficients of the polynomials) can be chosen real. It is clear that $W$ sends $G_{\mathbf{R}}$ to Poly $_{\mathbf{R}}^{2 d-2}$. The Wronski map is a finite regular map of compact algebraic manifolds, and its degree can be defined as the number of preimages of a generic point. This number turns out to be the Catalan number $u_{d}$, see, for example, 6]. If the Grassmannian $G$ is embedded in a projective space by the Plücker embedding, then the Wronski map $W$ becomes a restriction of a linear projection on $G$. Thus the degree of $W$ is the same as the degree of the Grassmann variety, that is the number of intersections of a generic subspace of codimension $2 d-2$ with the Plücker embedding of $G$.

Using this notation, theorems 1 and 2 can be restated as follows:

1. The full preimage of a polynomial with all real roots under the Wronski map consists of real points in $G$.

2. Every polynomial in Poly ${ }^{2 d-2}$ with distinct real roots has exactly $u_{d}$ distinct preimages under the Wronski map.

If a pair $\left(f_{1}, f_{2}\right)$ represents a point of $G$, then $g=f_{1} / f_{2}$ is a non-constant rational function of degree at most $d$. If two pairs of polynomials represent the same point of $G$, then the corresponding rational functions are equivalent. This defines a map $r$ from $G$ into the set $\operatorname{Rat}^{d}$ of equivalence classes of nonconstant rational functions of degree at most $d$. This map is not injective because polynomials in a pair can have a common factor. More precisely, let $Z_{0} \subset G$ be the locus of points corresponding to pairs of polynomials having a non-constant common factor, and $Z_{1}$ the locus corresponding to polynomials of degree less than $d$. Put $Z=Z_{0} \cup Z_{1}$. Then

$$
r: G \backslash Z_{0} \rightarrow \text { Rat }^{d}
$$

is a bijection. The standard topology on $G$ can be defined as induced by the Plücker embedding, and the topology on Rat ${ }^{d}$ is of uniform convergence with respect to the spherical metric. The map $r$ is continuous on $G \backslash Z$ but not continuous on the whole $G$. The following weaker continuity property of $r$ holds on the whole Grassmannian. 
Proposition 1. Let $\left(p_{j}\right)$ be a converging sequence in $G, p_{j} \in G \backslash Z$ and $p=\lim p_{j}$ is represented by a pair of polynomials with a common factor $q$. Let $z_{1}, \ldots, z_{k}$ be the roots of $q$. Then $r\left(p_{j}\right) \rightarrow r(p)$ uniformly on compact subsets of $\mathbf{C} \backslash\left\{z_{1}, \ldots, z_{k}, \infty\right\}$. One does not have to include $\infty$ if the degree of $p$ is the same as that of $p_{j}$.

The elementary proof is left to the reader.

\subsection{Nets of rational functions}

Let $R^{d}$ be the class of real non-constant rational functions $g$ of degree at most $d$ whose all critical points are real. Consider the full preimage $\gamma=g^{-1}(\overline{\mathbf{R}})$, where $\overline{\mathbf{R}}=\mathbf{R} \cup\{\infty\}$. This preimage consists of simple analytic arcs which meet only at critical points. These arcs define a cell decomposition $C(g)$ of the Riemann sphere $\mathbf{P}^{1}$ whose 2-cells (faces) are components of $\mathbf{P}^{1} \backslash \gamma, 1$ cells (edges) are components of $\gamma \backslash$ \{critical points $\}$ and 0-cells (vertices) are the critical points. We choose some vertex $v_{0}$ and call it the distinguished vertex of $C(g)$. The union $\gamma$ of edges and vertices is the 1 -skeleton of the cell decomposition. Such a cell decomposition $C=C(g)$ has the following properties:

(i) the 1-skeleton of $C$ contains $\overline{\mathbf{R}}$.

(ii) $C$ is symmetric with respect to $\overline{\mathbf{R}}$,

(iii) all vertices belong to $\overline{\mathbf{R}}$ and an even number of edges meet at each vertex.

If $C=C(g)$ the even number in (iii) is twice the local degree of $g$ at the critical point. Another important property of our cell decomposition is that the closure of every cell is homeomorphic to a closed ball of the same dimension. It follows that

(iv) no edge can begin and end at the same point.

Two cell decompositions $C_{1}$ and $C_{2}$ satisfying (i)-(iv) and having distinguished vertices $v_{0}^{1}$ and $v_{0}^{2}$ will be called equivalent if there exists a homeomorphism $\phi: \mathbf{P}^{1} \rightarrow \mathbf{P}^{1}$ commuting with reflection with respect to $\overline{\mathbf{R}}$, preserving orientations of $\overline{\mathbf{R}}$ and $\mathbf{P}^{1}$, mapping cells of $C_{1}$ onto cells of $C_{2}$ and $v_{0}^{1}$ to $v_{0}^{2}$. An equivalence class of cell decompositions satisfying (i)-(iv) will be called a net. The number of faces of a net is even, we denote it by $2 d$ and call the positive integer $d$ the degree of the net. If $C=C(g)$ then $\operatorname{deg} C=\operatorname{deg} g$. A 
net of degree $d$ has $2 d-2$ edges disjoint from the real axis. Using the Uniformization theorem, we proved in [1, 2, that each net comes from a rational function, and the critical points of this rational function can be arbitrarily prescribed, but we do not use this result here, and in fact it will be deduced from our theorems 1 and 2 in the end of Section 1.

We need the following elementary

Proposition 2. Let $\left(p_{j}\right)$ be a convergent sequence in $G_{\mathbf{R}}$, and $p=\lim p_{j}$. Let $g_{j}=r\left(p_{j}\right)$ be the corresponding sequence of rational functions. Then the sets $\gamma_{j}=g_{j}^{-1}(\overline{\mathbf{R}})$ converge in the Hausdorff metric to the set $\gamma=g^{-1}(\overline{\mathbf{R}})$, where $g=r(p)$.

This is a simple corollary of Proposition 1, and the details are left to the reader.

Corollary 1. Suppose that $g_{t}: t \in[0,1]$ is a continuous path in $R^{d}$ and each $g_{t}$ has $2 d-2$ simple critical points. Let one of these critical points be $v_{0}(t)$, a continuous function of $t$. Then the net of $g_{t}$ with distinguished point $v_{0}(t)$ is independent of $t$.

Corollary 2. Let $p_{t}$ be a continuous path in the Grassmannian $G$, parametrized by $[0,1]$. Suppose that $g_{t}=r\left(p_{t}\right)$ belong to $R^{d}$ and have critical points $x_{0}(t), \ldots, x_{n}(t)$, such that $x_{j}(t) \neq x_{i}(t)$ for $0 \leq j<i \leq n$ and $0 \leq t<1$, while for $t=1$ we have $x_{0}(1)=x_{1}(1)$ and $x_{j}(t) \neq x_{i}(t)$ for $1 \leq j<i \leq n$.

Then the degrees of $g_{t}$ are equal for $t \in[0,1)$, and the degree of $g_{1}$ is less than the degree of $g_{t}, t \in[0,1)$ if and only if the net of $g_{0}$ contains an edge from $x_{0}$ to $x_{1}$.

Proof. According to Proposition 2, the sets $\gamma_{t}=g_{t}^{-1}(\overline{\mathbf{R}})$ vary continuously in the Hausdorff metric. If there is an edge connecting $x_{0}(t)$ and $x_{1}(t)$, the limit of this edge as $t \rightarrow 1$ cannot be a loop because of the property (iv) of the nets. So the limit belongs to $\overline{\mathbf{R}}$ and thus the limit cell decomposition has fewer faces than the cell decomposition $C\left(g_{1}\right)$.

In the opposite direction, if this limit has fewer faces, some edge has to disappear in the limit, and this can only be an edge from $x_{0}$ to $x_{1}$.

\subsection{Thorns}

Let $X^{2 d-2} \subset$ Poly $_{\mathbf{R}}^{2 d-2}$ be the subset consisting of polynomials whose all roots are real. Then $X^{2 d-2}$ has non-empty interior. Here we construct an open 
subset of $X^{2 d-2}$ such that for every polynomial $p$ in this subset, the full preimage $W^{-1}(p)$ consists of $u_{d}$ distinct real points in $G$. The existence of such a subset was established by Sottile [14, but we give a more precise description of this set following [3].

We fix $d \geq 2$. Consider pairs of integers

$$
0 \leq k_{1}<k_{2} \leq d
$$

and pairs $\mathbf{q}=\left(q_{1}, q_{2}\right)$ of real polynomials

$$
\begin{array}{ll}
q_{1}(z)= & z^{d-1}+a_{1, d-2} z^{d-2}+\ldots+a_{1, k_{1}} z^{k_{1}}, \\
q_{2}(z)= & z^{d}+a_{2, d-1} z^{d-1}+\ldots+a_{2, k_{2}} z^{k_{2}} .
\end{array}
$$

Suppose that all coefficients $a_{i, j}$ are strictly positive, all roots of the Wronskian determinant $W(\mathbf{q})=W\left(q_{1}, q_{2}\right)$ belong to the semi-open interval $(-1,0] \subset \mathbf{R}$, and those roots on the open interval $(-1,0)$ are simple. The set of all such polynomial pairs (6) will be denoted by $b\left(k_{1}, k_{2}\right)$. The greatest common factor of $\left\{q_{1}, q_{2}\right\}$ is $z^{k_{1}}$.

It is easy to see that the representation of a point of $G_{\mathbf{R}}$ by a pair in $b\left(k_{1}, k_{2}\right)$ is unique. Setting $k_{1}=0$ and $k_{2}=1$ we obtain an open subset $b(0,1)$ of $G_{\mathbf{R}}$.

The multiplicity of the root of $W(\mathbf{q})$ at 0 is $k=k_{1}+k_{2}-1$. We enumerate the negative roots of $W(\mathbf{q})$ as

$$
-x_{2 d-2}<-x_{2 d-3}<\ldots<-x_{k+1}<0
$$

Let $\epsilon$ be a positive increasing function on $[0,1]$ satisfying $\epsilon(0)=0, \epsilon(x)<$ $x$, for $x \in(0,1]$. The set of all such functions will be denoted by $E$. A thorn $T$ of dimension $n$ is a region in $\mathbf{R}^{n}$ of the form

$$
T(n, \epsilon)=\left\{\left(y_{1}, \ldots, y_{n}\right): 0<y_{n}<\epsilon(1), 0<y_{k}<\epsilon\left(y_{k+1}\right), 1 \leq k \leq n-1\right\} .
$$

Let $w(k, T)$ be the set of polynomials of the form

$$
p(x)=x^{k}\left(x+x_{2 d-2}\right)\left(x+x_{2 d-3}\right) \ldots\left(x+x_{k+1}\right),
$$

where the vectors $\left(x_{2 d-2}, \ldots, x_{k+1}\right)$ belong to a thorn $T$ of dimension $2 d-2-k$.

The set $b(d-1, d)$ consists of a single pair $q_{1}=z^{d-1}, q_{2}=z^{d}$.

Consider the following two operations $F^{i}, i=1,2$. For each pair $\left(q_{1}, q_{2}\right)$, of the form (6) operation $F^{i}$ adds to the polynomial $q_{i}$ one term $a z^{k_{i}-1}$, 
where $a>0$ is a small parameter, and leaves the other polynomial of the pair unchanged. So each operation $F^{i}$ increases the total number of non-zero coefficients of a polynomial pair ([6) by one:

$$
F_{a}^{i}: b(\mathbf{k}) \rightarrow b\left(\mathbf{k}-\mathbf{e}_{i}\right), \quad F_{a}^{i}(\mathbf{q})=\mathbf{q}+a z^{k_{i}-1} \mathbf{e}_{i},
$$

where $\mathbf{k}=\left(k_{1}, k_{2}\right)$ and $\left(\mathbf{e}_{1}, \mathbf{e}_{2}\right)$ is the standard basis in $\mathbf{R}^{2}$, and $a>0$ is a small parameter whose range may depend on $\mathbf{q}$. The following rule has to be observed:

Rule. Operation $F^{i}$ is permitted on $b\left(k_{1}, k_{2}\right)$ if and only if the outcome of this operation does not violate inequalities (5).

In other words, operation $F^{1}$ is permitted on $b\left(k_{1}, k_{2}\right)$ if $k_{1}>0$, and operation $F^{2}$ is permitted on $b\left(k_{1}, k_{2}\right)$ if $k_{2}>k_{1}+1$.

The following result, which is a part of [3, Proposition 8] shows, among other things, that the $F^{i}$ are well defined, that is their result indeed belongs to some $b\left(k_{1}^{*}, k_{2}^{*}\right)$ for sufficiently small values of the parameter $a$.

Proposition 3. Suppose that $\mathbf{k}=\left(k_{1}, k_{2}\right)$ and $i \in\{1,2\}$ satisfy the Rule above. Suppose that for some thorn $T$ of dimension $2 d-2-k$ a set $U \subset$ $b\left(k_{1}, k_{2}\right)$ is given, such that the map $\mathbf{q} \mapsto W(\mathbf{q}): U \rightarrow w(k, T)$ is surjective. Then there exists a thorn $T^{*}$ of dimension $2 d-1-k$ and a set $U^{*} \subset b\left(\mathbf{k}-\mathbf{e}_{i}\right)$, such that every $\mathbf{q}^{*} \in U^{*}$ has the form $F_{a}^{i}(\mathbf{q}), \mathbf{q} \in U$, where $F_{a}^{i}$ is defined in (9), and $a>0$, and the map

$$
\mathbf{q}^{*} \mapsto W\left(\mathbf{q}^{*}\right): U^{*} \rightarrow w\left(k-1, T^{*}\right)
$$

is surjective.

Proof. We follow [3, Section 2]. First we state three elementary lemmas about thorns.

Lemma 1. Intersection of any finite set of thorns of same dimension contains a thorn of the same dimension.

Proof. Take the minimum of their defining functions.

Lemma 2. Let $T=T(n, \epsilon)$ be a thorn of dimension $n$ in $\mathbf{R}^{n}=\left\{\left(x_{1}, \ldots, x_{n}\right)\right\}$, and $U$ its neighborhood in $\mathbf{R}^{n+1}=\left\{\left(x_{0}, \ldots, x_{n}\right)\right\}$. Then $U^{+}=U \cap \mathbf{R}_{>0}^{n+1}$ contains a thorn $T\left(n+1, \epsilon_{1}\right)$.

Proof. There exists a continuous function $\delta_{0}: T \rightarrow \mathbf{R}_{>0}$, such that $U^{+}$ contains the set $\left\{\left(x_{0}, \mathbf{x}\right): \mathbf{x} \in T, 0<x_{0}<\delta_{0}(\mathbf{x})\right\}$. Let $\delta(t)$ be the minimum 
of $\delta_{0}$ on the compact subset $\left\{\mathbf{x} \in \overline{T(n, \epsilon / 2)}: x_{1} \geq t\right\}$ of $T$. Then there exists $\epsilon_{0} \in E$ with the property $\epsilon_{0}<\delta$. If we define $\epsilon_{1}=\min \left\{\epsilon / 2, \epsilon_{0}\right\}$, then $T\left(n+1, \epsilon_{1}\right) \subset U^{+}$.

Lemma 3. Let $T=T(n+1, \epsilon)$ be a thorn of dimension $n+1$, and $h: T \rightarrow \mathbf{R}_{>0}^{n+1},\left(x_{0}, \mathbf{x}\right) \mapsto\left(y_{0}\left(x_{0}, \mathbf{x}\right), \mathbf{y}\left(x_{0}, \mathbf{x}\right)\right)$, a continuous map with the properties: for every $\mathbf{x}$ such that $\left(x_{0}, \mathbf{x}\right) \in T$ for some $x_{0}>0$, the function $x_{0} \mapsto y_{0}\left(x_{0}, \mathbf{x}\right)$ is increasing, and $\lim _{x_{0} \rightarrow 0} \mathbf{y}\left(x_{0}, \mathbf{x}\right)=\mathbf{x}$. Then the image $h(T)$ contains a thorn.

Proof. We consider the region $D \in \mathbf{R}^{n+1}$ consisting of $T$, its reflection $T^{\prime}$ in the hyperplane $x_{0}=0$ and the interior with respect to this hyperplane of the common boundary of $T$ and $T^{\prime}$. The map $h$ extends to $T^{\prime}$ by symmetry: $h\left(-x_{0}, \mathbf{x}\right)=-h\left(x_{0}, \mathbf{x}\right),\left(x_{0}, \mathbf{x}\right) \in T$, and then to the whole $D$ by continuity. It is easy to see that the image of the extended map contains a neighborhood $U$ of the intersection of $D$ with the hyperplane $x_{0}=0$. This intersection is a thorn $T_{1}$ in $\mathbf{R}^{n}=\left\{\left(x_{0}, \mathbf{x}\right) \in \mathbf{R}^{n+1}: x_{0}=0\right\}$. Applying Lemma 2 to this thorn $T_{1}$, we conclude that $U^{+}$contains a thorn.

We continue the proof of Proposition 3.

Let us fix $\mathbf{q} \in U$, and put $W=W_{\mathbf{q}}$. As $W \in w(k, \epsilon)$, we have ord $W=k$, where ord denotes the multiplicity of a root at 0 . Let $c z^{k}$ be the term of the smallest degree in $W(z)$. Then $c>0$, because all roots of $W$ are non-positive. In fact,

$$
c=\left(k_{2}-k_{1}\right) a_{2, k_{2}} a_{1, k_{1}}>0 .
$$

We fix $i \in\{1,2\}$ satisfying the Rule above and define $W^{*}=W_{\mathbf{q}^{*}}$, where $\mathbf{q}^{*}=F_{a}^{i}(\mathbf{q})$. Then ord $W^{*}=k-1$ and the term of the smallest degree in $W^{*}(z)$ is $c^{*} z^{k-1}$, where

$$
c^{*}=a\left(k_{2}^{*}-k_{1}^{*}\right) a_{3-i, k_{3-i}}>0,
$$

We conclude that when $a$ is small enough (depending on $\mathbf{q}$ ), the Wronskian $W^{*}$ has one simple root in a neighborhood of each negative root of $W$, and in addition, one simple negative root close to zero, and a root of multiplicity $k-1$ at 0 . To make this more precise, we denote the negative roots of $W$ and $W^{*}$ by

$$
-x_{2 d-2}<\ldots<-x_{k+1} \text { and }-y_{2 d-2}<\ldots<-y_{k+1}<-y_{k}
$$

where $y_{j}=y_{j}(a)$. We have

$$
y_{j}(0)=x_{j}, \quad \text { for } \quad 1 \leq j \leq n, \quad \text { and } \quad y_{k}(0)=0 .
$$


Furthermore, if $a$ is small enough (depending on $\mathbf{q}$ )

$$
a \mapsto y_{k}(a) \text { is increasing and continuous. }
$$

The set $w(k, \epsilon)$ is parametrized by a thorn $T=T(2 d-2-k, \epsilon)$, where $\mathbf{x}=\left(x_{k+1}, \ldots, x_{2 d-2}\right)$. There exists a continuous function $\delta: T \rightarrow \mathbf{R}_{>0}$, such that

$$
\mathbf{q}^{*} \in b\left(\mathbf{k}^{*}\right), \quad \text { for } \quad a \in(0, \delta(\mathbf{x})), \quad \mathbf{x} \in T .
$$

It remains to achieve (10) by modifying the thorn $T$. Consider the set

$$
U^{*}=\left\{\mathbf{q}^{*}=F_{a}\left(\mathbf{q}_{\mathbf{x}}\right): \mathbf{x} \in T, a \in(0, \delta(\mathbf{x}))\right\} \subset b\left(\mathbf{k}^{*}\right),
$$

where $\mathbf{q}_{\mathbf{x}} \in U$ is some preimage under $W$ of the polynomial (8) with $\left(x_{k+1}, \ldots, x_{2 d-2}\right)=\mathbf{x}$. Such preimage exists by assumption of Proposition 3 that the map $\mathbf{q} \mapsto W_{\mathbf{q}}, U \rightarrow w(k, T)$ is surjective. We apply Lemma 2 to the half-neighborhood (17) of $T$, with $x_{k}=a$, to obtain a thorn $T_{1}\left(2 d-k-1, \epsilon_{1}\right)$. Then we apply Lemma 3 to the map $h: T_{1} \rightarrow \mathbf{R}_{>0}^{2 d-k-1}$, defined by $y_{j}=$ $y_{j}\left(x_{0}, \mathbf{x}\right)$, where $y_{j}$ are as in (13), and $x_{k}=a$.

This map $h$ satisfies all conditions of Lemma 3 in view of (14) and (15). This proves (10) and Proposition 3.

We begin with the single element of $b(d-1, d)$ and apply operations $F^{i}$ in some sequence, obeying the Rule above, while possible. As every step decreases $k$ by 1 , the total number of steps will be $2 d-2$. We describe the sequence of steps by a sequence $\sigma$ of 1's and 2's of length $2 d-2$. The number $i$ on the $n$-th place in this sequence indicates that operator $F^{i}$ was applied on $n$-th step. The Rule above translates to the following characterization of all possible sequences $\sigma$ :

a) The numbers of 1's and 2's in $\sigma$ are equal.

b) In each initial segment of $\sigma$ the number of 1's is not less than the number of 2's.

Such sequences are called ballot sequences (for two candidates), see, for example, [17]. The number of ballot sequences of length $2 d-2$ is the Catalan number $u_{d}$.

Applying $2 d-2$ times Proposition 3 according to each ballot sequence $\sigma$ we obtain in the end an open subset $U_{\sigma} \subset b(0,1)$ which is mapped surjectively by the Wronski map onto $w(0, T)$ for some thorn $T$. As the intersection of any finite set of thorns of the same dimension contains a thorn of the same 
dimension by Lemma 1, we may assume that this set $w(0, T)$ is the same for all sequences $\sigma$.

When applying Proposition 3 we can make the range of parameter $a$ as small as desired; using this we can assure that the sequence of coefficients of the pair (6) is monotone: the coefficients decrease in the order of their appearance. This implies that the sets $U_{\sigma}$ with different $\sigma$ are disjoint.

So we obtain $u_{d}$ disjoint open sets $U_{\sigma}$, and each of them is mapped onto $w(0, T)$ continuously and surjectively by the Wronski map $W$. As the number of preimages of any point under $W$ is at most $u_{d}$ we conclude that the maps $W: U_{\sigma} \rightarrow w(0, T)$ are homeomorphisms for all $\sigma$.

Thus each point of the open subset $w(0, T) \subset X^{2 d-2}$ has exactly $u_{d}$ preimages under $W$ and all these preimages are real. Each of these preimages cor-

responds to an analytic branch of the inverse $W^{-1}$ on $w(0, T)$. The branches are enumerated by ballot sequences.

\subsection{Completion of the proof}

Let us fix a thorn $T$ such that each polynomial in $w(0, T)$ has $u_{d}$ different real preimages under the Wronski map, as in the end of the previous section.

To each of these preimages $\mathbf{q}=\left(q_{1}, q_{2}\right)$ corresponds a rational function $r(\mathbf{q})=q_{1} / q_{2}$ in $R^{d}$ with $2 d-2$ distinct real critical points which has a net $\gamma(\mathbf{q})$. We take the rightmost critical point of these functions as distinguished vertices of the nets.

We claim that all these $u_{d}$ nets are different. To prove the claim we just show how to determine the net from the ballot sequence and vice versa.

Proposition 4. Let $k=0$, and let $p$ be a polynomial in $w(0, T)$ of the form (8). Let $\mathbf{q}=\left(q_{1}, q_{2}\right)$ be a polynomial pair as in (6) corresponding to a point in $W^{-1}(p)$, with the ballot sequence $\sigma$, and $g=r(\mathbf{q})=q_{1} / q_{2}$.

Then the net of $g$ contains an edge between $x_{m}$ and $x_{m+1}$ if and only if the $m$-th member of the sequence $\sigma$ is 1 .

Proof. It is enough to investigate what happens to a net when an operator $F^{i}$ of Proposition 3 is applied. We see from (6) and (96) that the degree of $q_{1} / q_{2}$ increases if and only if $i=1$. Corollary 2 of Proposition 2 says that this happens if and only if the net has an edge between $x_{m}$ and $x_{m+1}$. This proves Proposition 4.

So we obtained a polynomial $p_{0} \in w(0, T)$ of degree $2 d-2$ with $2 d-2$ 
real roots whose preimage under the Wronski map consists of $u_{d}$ pairs with different nets. Each of these preimages corresponds to a holomorphic inverse branch of the Wronski map in $w(0, T)$. Let $p_{1}$ be any real polynomial of degree $2 d-2$ with $2 d-2$ real roots. Then there exists a path $p_{t}: t \in[0,1]$ in $X^{2 d-2}$ connecting $p_{0}$ and $p_{1}$, such that all $p_{t}$ are polynomials with $2 d-2$ distinct roots. For example one can connect the corresponding roots of $p_{0}$ and $p_{1}$ linearly. We do analytic continuation of all the inverse branches of the Wronski map along this path. As critical points of our rational functions cannot collide (because the zeros of their Wronskians $p_{t}$ do not collide), their nets do not change under the continuation. Suppose that this analytic continuation to $t=1$ is impossible. Let $t_{0}$ be the smallest singular point. Then $p_{t_{0}}$ is a ramified value of the Wronski map and the full preimage $W^{-1}\left(p_{t_{0}}\right)$ consists of fewer than $u_{d}$ points. This full preimage still consists of real rational functions with all critical points real and distinct, so the nets are defined for all elements of this preimage. This means that at least two oneparametric families of rational functions with different nets tend to the same function with $2 d-2$ distinct critical points, which is impossible by Corollary 1 of Proposition 2.

This proves theorems 1 and 2 .

This proof clearly implies that for any given net there exists a unique class of real rational functions with all critical points real, and the critical points of these functions can be chosen arbitrarily, the result which was established in [1] with the help of the Uniformization theorem and rather complicated topological considerations.

To prove Theorem 3, we notice that 1-skeleton of every net of degree $d$ can be obtained as the limit of 1 -skeletons of nets with $2 d-2$ vertices. So every net of degree $d$ actually occurs as a net of a real rational function of the class of degree $d$ with all critical points real. Counting the nets of degree $d$ with $q$ vertices of degrees $2 a_{1} \ldots, 2 a_{q}$ gives the Kostka number $K_{\mathbf{a}}$ (see, for example, [5, Lemma 3]. So there are at least $K_{\mathbf{a}}$ classes of rational functions of degree $d$ with prescribed real critical points. On the other hand, Schubert calculus [11] shows that there are at most $K_{\mathbf{a}}$ classes of rational functions with any prescribed critical points of multiplicities $a_{1}, \ldots, a_{q}$. This proves Theorem 3.

Corollary. To each net of degree d corresponds exactly one class of real rational functions of degree d with prescribed real critical points. 


\section{Fuchsian equations}

\subsection{Equations with all polynomial solutions}

Suppose that distinct points $a_{1}, \ldots, a_{n}$ in the complex plane are given. We want to describe the set of equivalence classes of polynomial pairs $\left(y_{1}, y_{2}\right)$, such that

$$
W\left(y_{1}, y_{2}\right) \sim\left(z-a_{1}\right) \ldots\left(z-a_{n}\right) .
$$

This time we do not specify in advance the degree of polynomials $y_{1}, y_{2}$ but it is easy to see that it is at most $n+1$. As $W\left(y_{1}, y_{2}\right)$ has only simple zeros, the polynomials $y_{1}$ and $y_{2}$ are co-prime.

To approach this problem, we introduce a special parametrization of equivalence classes of polynomial pairs whose Wronskian has prescribed zeros. Recall that for any two linearly independent functions $y_{1}$ and $y_{2}$ one can write a second order linear differential equation which has these two functions as solutions:

$$
\left|\begin{array}{ccc}
y & y_{1} & y_{2} \\
y^{\prime} & y_{1}^{\prime} & y_{2}^{\prime} \\
y^{\prime \prime} & y_{1}^{\prime \prime} & y_{2}^{\prime \prime}
\end{array}\right|=A y^{\prime \prime}+B y^{\prime}+C y=0
$$

where

$$
A=W\left(y_{1}, y_{2}\right), \quad B=-A^{\prime} \quad \text { and } \quad C=W\left(y_{1}^{\prime}, y_{2}^{\prime}\right) .
$$

If $y_{1}$ and $y_{2}$ are polynomials, then $A, B, C$ are also polynomials, and we have

$$
\operatorname{deg} B \leq \operatorname{deg} A-1, \quad \operatorname{deg} C \leq \operatorname{deg} A-2 .
$$

These conditions are equivalent to regularity of the singular point at infinity. Introducing two rational functions $P=B / A$ and $Q=C / A$, we conclude that $P(\infty)=Q(\infty)=0$. Furthermore, $P=-A^{\prime} / A$, and $A$ has only simple zeros, so all residues of $P$ in $\mathbf{C}$ are equal to -1 . Denoting the residues of $Q$ by $x_{j}$, we obtain

$$
P(z)=-\sum_{j=1}^{n} \frac{1}{z-a_{j}} \quad \text { and } \quad Q(z)=\sum_{j=1}^{n} \frac{x_{j}}{z-a_{j}} .
$$

Evidently, $P, Q$ and $x_{j}$ depend only on the equivalence class of the polynomial pair $\left(y_{1}, y_{2}\right)$. 
Now we write the conditions on $x_{j}$ which express the fact that all solutions of the differential equation

$$
y^{\prime \prime}+P y^{\prime}+Q y=0
$$

are polynomials.

In a neighborhood of a singular point $a_{k}$, our equation can be written in the form:

$$
\left(z-a_{k}\right) y^{\prime \prime}+P_{k}(z) y^{\prime}+Q_{k}(z) y=0,
$$

with

$$
P_{k}(z)=-1+p_{k}\left(z-a_{k}\right)+O\left(z-a_{k}\right)^{2}, \quad \text { where } \quad p_{k}=\sum_{j \neq k} \frac{1}{a_{j}-a_{k}},
$$

and

$$
Q_{k}(z)=x_{k}+q_{k}\left(z-a_{k}\right)+O\left(z-a_{k}\right)^{2}, \quad \text { where } \quad q_{k}=-\sum_{j \neq k} \frac{x_{j}}{a_{j}-a_{k}} .
$$

Our differential equation (22) has two linearly independent polynomial solutions without a common factor, so it has a polynomial solution of the form

$$
y(z)=1+c_{1}\left(z-a_{k}\right)+c_{2}\left(z-a_{k}\right)^{2}+O\left(z-a_{k}\right)^{3} .
$$

Differentiating this, we obtain

$$
\begin{gathered}
y^{\prime}(z)=c_{1}+2 c_{2}\left(z-a_{k}\right)+O\left(z-a_{k}\right)^{2}, \\
y^{\prime \prime}(z)=2 c_{2}+O\left(z-a_{k}\right) .
\end{gathered}
$$

Substituting this to (22), we obtain

$$
c_{1}=x_{k},
$$

and

$$
p_{k} c_{1}+x_{k} c_{1}+q_{k}=0
$$

SO

$$
x_{k}^{2}=-p_{k} x_{k}-q_{k} .
$$


Recalling the expressions for $p_{k}, q_{k}$, we obtain the following necessary condition for the equation (22) to have two linearly independent polynomial solutions:

$$
x_{k}^{2}=\sum_{j \neq k} \frac{x_{j}-x_{k}}{a_{j}-a_{k}}, \quad k=1, \ldots, n .
$$

Proposition 5. Condition (24) is necessary and sufficient for a differential equation (21) with coefficients (20) to have two linearly independent polynomial solutions.

Proof. It remains to prove sufficiency. From (22) we conclude that all singular points in $\mathbf{C}$ are regular, with exponents 0 and 2. Condition (23) guarantees that there is a power series solution corresponding to the smaller exponent. This implies that there are two linearly independent holomorphic solutions in a neighborhood of each singular point. Thus all solutions are entire functions. By a theorem of Halphen which can be found in 7 , 15.5], if the general solution of the equation (18), where $A, B, C$ satisfy $\operatorname{deg} B \leq \operatorname{deg} A, \operatorname{deg} C \leq \operatorname{deg} A$, is a meromorphic function in $\mathbf{C}$, then this meromorphic function has to be of the form

$$
\sum R_{j}(z) e^{\lambda_{j} z}
$$

where $R_{j}$ are rational functions. But asymptotics at infinity shows that under the stronger condition (19) on the degrees of the coefficients, the exponentials cannot be present. So the general solution is an entire rational function, that is a polynomial.

Proposition 6. Every solution of the system (24) has the following properties:

$$
\begin{gathered}
\sum_{k=1}^{n} x_{k}=0, \\
(n+1)^{2}-4 \sum_{k=1}^{n} x_{k} a_{k}=s^{2},
\end{gathered}
$$

where $s$ is an integer such that $n+s$ is odd, and $1 \leq s \leq n+1$.

This integer $s$ is the local degree at infinity of the rational function which is the ratio of two linearly independent solutions of (21) with $P$ and $Q$ as in (201). 
Proof of Proposition 6. By Proposition 5, for every solution $x_{1}, \ldots, x_{n}$ of (24), all solutions of the differential equation (21) are polynomials. Suppose that

$$
P(z)=p z^{-1}+O\left(z^{-2}\right), \quad Q(z)=q z^{-1}+q^{*} z^{-2}+O\left(z^{-3}\right) \quad z \rightarrow \infty .
$$

Then

$$
p=-n, \quad q=\sum_{k=1}^{n} x_{k} \quad \text { and } \quad q^{*}=\sum_{k=1}^{n} x_{k} a_{k} .
$$

Substituting into (21) a polynomial

$$
y(z)=z^{d}+\ldots,
$$

we first obtain

$$
q z^{d-1}+O\left(z^{d-2}\right)=0, \quad z \rightarrow \infty,
$$

so $q=0$ which proves (25). Then we obtain

$$
d(d-1) z^{d-2}+d p z^{d-2}+q^{*} z^{d-2}+O\left(z^{d-3}\right)=0,
$$

SO

$$
d^{2}-d(n+1)+q^{*}=0 .
$$

This equation has two solutions, the possible degrees of polynomials:

$$
d_{1,2}=\frac{n+1 \pm \sqrt{(n+1)^{2}-4 q^{*}}}{2}
$$

As all solutions of our differential equation are polynomials, there are two solutions of different degrees. This implies that both $d_{1}$ and $d_{2}$ are integers which implies (26). Notice that $d_{1}-d_{2}=s$.

Rational function $y_{1} / y_{2}$ is locally $s$ to 1 at infinity, from which the inequality $1 \leq s \leq n+1$ follows.

So our theorems 1 and 2 are can be restated as

Theorem 4. If all $a_{k}$ are real then all solutions of (24) are real. Moreover, each solution $\left(x_{1}, \ldots, x_{n}\right)$ can be analytically continued as a function of parameters $\left(a_{1}, \ldots, a_{n}\right)$ in the region $a_{1}<a_{2}<\ldots<a_{n}$.

Solutions $\left(x_{1}, \ldots, x_{n}\right)$ correspond to classes of rational functions of all degrees $d \in[n / 2+1, n+1]$ if $n$ is even and $d \in[(n+1) / 2+1, n+1]$ if 
$n$ is odd, having simple critical points at $a_{1}, \ldots, a_{n}$ and possibly a critical point at infinity. The order of the critical point at infinity is $s-1$ where $s$ is defined in (26).

System (24) has a trivial solution $x_{1}=\ldots=x_{n}=0$ which corresponds to the polynomial of degree $n+1$ with critical points $a_{1}, \ldots, a_{n}$. The opposite case is that $s=1$ (then $n$ is even) and we have rational functions with $n$ prescribed simple critical points. This case is characterized by the condition

$$
q^{*}=\left(n^{2}+2 n\right) / 4
$$

System (24) can be easily generalized to $p$-tuples of linearly independent polynomials satisfying a Fuchsian differential equation of order $p$. An analog of Theorem 4 for this case would be equivalent to the Shapiro conjecture for $p$ polynomials. For example, for $p=3$ one obtains the following system of equations with respect to $x_{k}$ and $u_{k}$ :

$$
\begin{aligned}
x_{k}^{2} & =\sum_{j \neq k} \frac{x_{j}-x_{k}}{a_{j}-a_{k}}-u_{k}, \\
x_{k} u_{k} & =\sum_{j \neq k} \frac{u_{j}-u_{k}}{a_{j}-a_{k}} .
\end{aligned}
$$

\subsection{Equilibria of electric charges in the plane}

Following Stieltjes [18, we state an extremal problem of potential theory which is equivalent to (24).

Suppose as above that

$$
A(z)=\left(z-a_{1}\right) \cdots\left(z-a_{n}\right), \quad \text { and } \quad B=-A^{\prime}
$$

in (18), and let us look for polynomials $C$ such that (18) has a polynomial solution

$$
y(z)=\left(z-z_{1}\right) \ldots\left(z-z_{m}\right) \quad \text { with } \quad z_{j} \in \mathbf{C} \backslash\left\{a_{1}, \ldots, a_{n}\right\} .
$$

We substitute (28) in (18) and put $z=z_{k}$ :

$$
A\left(z_{k}\right) y^{\prime \prime}\left(z_{k}\right)-A^{\prime}\left(z_{k}\right) y^{\prime}\left(z_{k}\right)=0,
$$

or

$$
y^{\prime \prime}\left(z_{k}\right) / y^{\prime}\left(z_{k}\right)-A^{\prime}\left(z_{k}\right) / A\left(z_{k}\right)=0,
$$


which is equivalent to

$$
2 \sum_{j \neq k} \frac{1}{z_{k}-z_{j}}-\sum_{j=1}^{n} \frac{1}{z_{k}-a_{j}}=0 .
$$

Equation (29) has the following physical interpretation. Positive unit charges +1 are fixed at the points $a_{k}$, and negatively charged particles of charge -2 each at the points $z_{k}$ are allowed to move in the plane. The particles are repelled or attracted according to their charges, and the force is inverse proportional to the distance ${ }^{1}$. So the force between $z$ and $w$ is $c /(\overline{z-w})$, where the bar stands for the complex conjugation and $c$ is a real constant depending on the charges. Then the equilibrium condition is expressed by the system of equations (29). The energy of such configuration is

$$
E=\log \frac{\prod_{(j, k): j \neq k}\left|z_{k}-z_{j}\right|^{2}}{\prod_{(j, k)}\left|z_{k}-a_{j}\right|} .
$$

The function $U(\mathbf{z}, \mathbf{a})$ under the logarithm is called the master function in 19, 9, 11, 12. Here $\mathbf{z}=\left(z_{1}, \ldots, z_{m}\right)$ is the variable and $\mathbf{a}=\left(a_{1}, \ldots, a_{n}\right)$ the parameter. Equilibrium configurations are the critical points of the master function. It is evident from physical considerations and easy to prove that all these equilibria are unstable.

So for a given polynomial $A$ as in (27), an equation of the form

$$
A y^{\prime \prime}-A^{\prime} y^{\prime}+C y=0
$$

has a non-trivial polynomial solution $y$ if and only if

$$
C=\left(-A y^{\prime \prime}+A^{\prime} y^{\prime}\right) / y,
$$

with $y$ given by (28) and $\left(z_{1}, \ldots, z_{m}\right)$ satisfying (29))

Lemma 4. If the equation (30) has one non-trivial polynomial solution, then all its solutions are polynomials.

This is [19, Lemma 7]. We include a proof for the reader's convenience.

\footnotetext{
${ }^{1}$ The particles in the plane can be imagined as infinite uniformly charged wires perpendicular to the complex plane and interacting by the Coulomb law.
} 
Proof. We apply the usual method of finding a second linearly independent solution when one, say $y_{1}$, is given. We obtain

$$
y_{2}=y_{1} \int A y_{1}^{-2}
$$

This can have ramification points only at the zeros of $y_{1}$. But as a solution of a differential equation (30), $y_{2}$ can only have ramification points at the zeros of $A$. As these two sets are disjoint, $y_{2}$ is an entire function. Now, using the expression (31) we conclude that $y_{2}$ is a polynomial. So all solutions are polynomials.

Remark. An alternative way to derive (29) is to write the condition that all residues of the integral in (31) are equal to zero.

We conclude that some solutions of (29) are not isolated; they occur in complex 1-dimensional families corresponding to configurations of zeros of all polynomial solutions of (30). However, each two-dimensional space of polynomials contains a one-dimensional subspace consisting of polynomials of smaller degree than generic polynomials in this space. Such polynomial of the smallest degree gives an isolated solution of (29). If $n=2 d-2$ and $m \leq d-1$, we see from (31) that $\operatorname{deg} y_{2}>\operatorname{deg} y_{1}$, and we conclude that the critical points of the master function $E$ are all isolated in this case. These facts about critical points of the master function and their relation to Fuchsian equations with polynomial solutions were discovered in [19]. They can be generalized to Fuchsian equation of arbitrary order. The equation (29) is a special case of the "Bethe Ansatz equation" in [19].

Suppose now that (30) has a polynomial solution. Then all solutions are polynomials and the ratio of any two linearly independent solutions is a rational function with all critical points real. Such a rational function is equivalent to a real rational function by Theorem 1, so we can find two real linearly independent solutions. Then $C$ is a real polynomial, and the solution of smallest degree is proportional to a real polynomial. Thus Theorem 1 has the following consequence:

Theorem 5. For given real points $a_{1}, \ldots, a_{n}$, each isolated equilibrium configuration $z_{1}, \ldots, z_{m}$ in (29) is symmetric with respect to the real line.

It is also easy to deduce Theorem 1 from Theorem 5 . 


\section{References}

[1] A. Eremenko and A. Gabrielov, Rational functions with real critical points and the B. and M. Shapiro conjecture in real enumerative geometry, Ann. Math. 155 (2002) 105-129.

[2] A. Eremenko and A. Gabrielov, Wronski map and Grassmannians of real codimension 2 subspaces, Computational Methods and Function Theory 1 (2001) 1-25.

[3] A. Eremenko and A. Gabrielov, Degrees of real Wronski maps, Discrete and Computational Geom. 28 (2002) 331-347.

[4] A. Eremenko and A. Gabrielov, Pole placement by static output feedback for generic linear systems, SIAM J. on Control and Opt. 41, 1 (2002) 303312 .

[5] A. Eremenko, A. Gabrielov, M. Shapiro and A. Vainshtein, Rational functions and real Schubert calculus, Proc. AMS (electronically published on July 25, 2005)

[6] L. Goldberg, Catalan numbers and branched coverings by the Riemann sphere. Adv. Math. 85, 2 (1991) 129-144.

[7] E. Ince, Ordinary Differential Equations, Longmans, Green and Co., London, 1927.

[8] V. Kharlamov and F. Sottile, Maximally inflected real rational curves, Mosc. Math. J. 3, 3 (2003) 947-987, 1199-1200.

[9] E. Mukhin and A. Varchenko, Critical points of master functions and flag varieties, Commun. Contemp. Math. 6, 1 (2004) 111-163.

[10] J. Rosenthal and F. Sottile, Some remarks on real and complex output feedback, Systems Control Lett. 33, 2 (1998) 73-80.

[11] I. Scherbak, Rational functions with prescribed critical points, Geom. Funct. Anal. 12, 6 (2002) 1365-1380.

[12] I. Scherbak, Intersections of Schubert varieties and critical points of the generating function, J. London Math. Soc. (2) 70, 3 (2004) 625-642. 
[13] V. Sedykh and B. Shapiro, On two conjectures concerning convex curves, to appear in Intl. J. Math.

[14] F. Sottile, The special Schubert calculus is real, Electron. Res. Announc. Amer. Math. Soc. 5 (1999), 35-39.

[15] F. Sottile, Enumerative real algebraic geometry, in: S. Basu and L. Gonzalez-Vega, Algorithmic and quantitative real algebraic geometry, 139-179, Amer. Math. Soc., Providence, RI, 2003.

[16] F. Sottile, Real Schubert calculus: polynomial systems and a conjecture of Shapiro and Shapiro, Experiment. Math. 9, 2 (2000) 161-182.

[17] R. Stanley, Enumerative combinatorics. Vol. 2, Cambridge University Press, Cambridge, 1999.

[18] T. Stieltjes, Sur certains polynômes qui vérifient une équation differentielle linéaire du second ordre et sur la théorie des fonctions de Lamé, Acta Math. 6 (1885) 321-326; Oeuvres complétes - Vol. 1, Springer, Berlin, 1993, 434-439.

[19] A. Varchenko and I. Scherbak, Critical points of functions, $\mathfrak{s l}_{2}$ representations, and Fuchsian differential equations with only univalued solutions. Mosc. Math. J. 3, 2 (2003) 621-645, 745.

Purdue University, West Lafayette, IN 47907

eremenko@math.purdue.edu

agabriel@math.purdue.edu 